\title{
Integrability and exact solution of correlated hopping multi-chain electron systems
}

\author{
Anjan Kundu* \\ Saha Institute of Nuclear Physics, 1/AF Bidhan Nagar, \\ 700064 Calcutta, India.
}

November 20, 2018

\begin{abstract}
Exact quantum integrability is established for a class of multi-chain electron models with correlated hopping and spin models with interchain interactions, by constructing the related Lax operators and $R$-matrices through twisting and gauge transformations. Exact solution of the eigenvalue problem for commuting conserved quantities of such systems is achieved through algebraic Bethe ansatz, on the examples of Hubbard and $t-J$ models with correlated hopping. Our systematic construction identifies the integrable subclass of such known solvable models and also generates new systems including the generalized $t-J$ models. At the same time it makes proper correction to a well known model and resolves recent controversies regarding the equivalence and solvability of some known models.
\end{abstract}

PACS: 02.30.IK, 04.20.Jb, 71.10.Pm, 05.30.Pr

Keywords: Quantum integrable systems, Algebraic Bethe ansatz, Gauge and twisting transformations, Correlated hopping electron and spin models

\section{Introduction}

There is an upsurge of interest in recent years in the study of correlated electron and spin systems in low dimensions, which is motivated mostly by the recent possibility of their fabrication and experimental verification of related theoretical results [1], as well as by their potential for

*e-mail: anjan@tnp.saha.ernet.in

${ }^{\dagger}$ Address during Sept.-Dec., 2001 : Inst. Theor. Phys., Uni-Dortmund. Germany 
applications to the high $T_{c}$-superconductivity [2]. On the other hand one can take an exclusive advantage in one-dimension, since some class of models in this case may become exactly solvable and even completely integrable, which therefore can enhance our understanding of the low-dimensional physics by providing detailed picture of the system through exact results. Few well known examples of such models are the $X X X$ and $X X Z$ spin chains [3], the Hubbard model [4, 5], the $t-J$ model [6, 7] etc., along with their various extensions and the ladder or their multi-chain generalisations [8, 9, 10]. Recently, a large class of models describing electrons with correlated hopping, spin ladders with interchain interactions and their multi-chain generalizations have attracted special attention due to their simplicity and exact solvability [11, 12, 13, 14, 15]. A systematic analysis has also been carried out [15 to identify the general class of these models which exhibits exact solvability by coordinate Bethe ansatz (CBA). However, the important question of complete quantum integrability for such systems, which defines a much richer class of models allowing infinite set of commuting conserved operators along with their exact eigenvalue solution through the algebraic Bethe ansatz (ABA) [16, has not been explored. Moreover, the recent controversy around the solvability [15] as well as the claim and denial of the equivalence between some models [17, 18] could not be solved satisfactorily due to the lack of understanding of the general structures for such systems.

Recall that the quantum integrability for a lattice model of $N$ sites ensures that, there must exist independent and mutually commuting set of conserved operators $\left\{C_{j}\right\}, j=1, \ldots, N$, the Hamiltonian being just one of them. For achieving this one usually shows that a one-parameter family of transfer-matrix $\tau(\lambda)=\operatorname{tr}\left(\prod_{j} L_{j}(\lambda)\right.$, constructed from the Lax operator $L_{j}(\lambda)$ and generating the set of conserved operators, themselves commute. This in turn follows from the Yang-Baxter equation (YBE) satisfied by the associated $L_{j}(\lambda)$-operator and the $R$-matrix solutions. Note that, while in CBA solvable models one can solve the eigenvalue problem (EVP) only for the Hamiltonian, the quantum integrable systems allow exact solution of the EVP, simultaneously for the whole set of conserved operators, including the Hamiltonian. This is done by solving the EVP of the transfer matrix: $\tau(\lambda)\left|n>=\Lambda_{n}(\lambda)\right| n>$ through ABA. Consequently, for describing quantum integrable systems [16] one has to start not from the Hamiltonian of the model but from the related solutions of the YBE.

Our aim here is to look into the class of solvable electron and spin models mentioned above, from the view point of quantum integrability. We therefore start by discovering the associated $R$-matrices and the quantum Lax operators for the integrable class in such systems. In doing so we exploit certain symmetries of the YBE under twisting and gauge transformations. This establishes in one hand the complete quantum integrability of the system and allows to apply the ABA method for exactly solving the EVP for the transfer matrix and consequently for all the conserved operators, simultaneously. At the same time our formulation helps to identify the 
integrable subclass of CBA solvable models classified in [15 and find the precise relationship between various existing models [11, 14, 13, 15] at their Lax operator as well as Hamiltonian level. As an important consequence, we detect some error in the derivation of the Zvyagin et al model [11, 12, derive the correct one and thus completely resolve the controversy regarding the solvability of the Zvyagin et al model and its equivalence with the Schulz-Shastry model [17, 18]. In addition our approach based on the construction of Lax-operators allows us to generate new quantum integrable models including generalized $t-J$ models

The organization of our paper is as follows. Sect. 2. describes briefly the main ideas of quantum integrability and ABA. Sect. 3 introduces the symmetries of YBE and the construction of the $R$-matrix and the Lax-operator. Sect. 4 is devoted to the derivation of models showing their mutual relations and establishes the equivalence between different models. The extension for our formulation to the multi-chain models and their equivalence are also considered here. Sect. 5 generates a new class of models including one for the generalised $t-J$ models. Sect. 6 presents the exact ABA solutions for the models constructed here. Sect. 7 is the concluding section.

\section{Quantum integrability, conserved operators and ABA}

By quantum integrability we will mean the integrability in the Liouville sense. For classical models such integrability means the existence of action-angle variables, when conserved quantities including the Hamiltonian can be expressed through action variables only. For quantum models, Liouville integrability similarly demands existence of sufficient number of mutually commuting conserved operators $\left\{C_{j}\right\}$, the Hamiltonian being one of them. The aim of the quantum inverse scattering is to solve the eigenvalue problem for all these conserved quantities, simultaneously. Recall in this context that the CBA is designed to solve this problem only for the Hamiltonian of the model. Therefore quantum integrable systems are much richer, though more involved and demanding than the CBA solvable models and as a rule the quantum integrability does not follow from its solvability by CBA. For describing integrable systems, naturally one can not start from the Hamiltonian as in CBA, but has to introduce some abstract objects like the Lax operator $L_{j}(\lambda)$ and the $R$-matrix, which depend on an extra parameter $\lambda$ called the spectral parameter [16]. Though the Lax operator is also a matrix similar to the $R$-matrix, it is a $p \times p$-matrix, while the matrix dimension of $R$ is $p^{2} \times p^{2}$. Another important difference is that, the elements of matrix $L_{j}(\lambda)$ are quantum operators defined at site $j$ and acting in the corresponding Hilbert space, while the elements of the $R$-matrix are the usual $c$-numbers. The matrices are considered here in the fundamental representation of the underlying algebra with its rank generally coinciding with $p-1$. For example for $s u(2)$, as in case of the spin- $\frac{1}{2}$ chains, 
the Lax operator would be a $2 \times 2$ and the $R$-matrix a $4 \times 4$ matrix.

A sufficient condition for the quantum integrability of the system may be given by the celebrated YBE

$$
R_{a b}(\lambda, \mu) L_{a j}(\lambda) L_{b j}(\mu)=L_{b j}(\mu) L_{a j}(\lambda) R_{a b}(\lambda, \mu), \quad j=1,2, \ldots, N
$$

where $a, b$ indicate the matrix or the auxiliary spaces, while $j$ the quantum spaces. The associativity property of the algebraic relation (2.1) yields in turn a similar relation

$$
R_{a b}(\lambda, \mu) R_{a c}(\lambda, \gamma) R_{b c}(\mu, \gamma)=R_{b c}(\mu, \gamma) R_{a c}(\lambda, \gamma) R_{a b}(\lambda, \mu)
$$

representing a compatibility condition for the $R$-matrix. The $R(\lambda, \mu)$-matrix usually depends on the difference between spectral parameters $\lambda$ and $\mu$, though there may be exceptions as in the case of Hubbard model. The well known and well studied integrable models belong to the so called ultralocal class, which along with the YBE (2.1) obeys also an additional restriction on the $L$-operators: $\left[L_{a i}(\lambda), L_{b j}(\mu)\right]=0, i \neq j, a \neq b$. Since the notion of integrability is intrinsically related to the conserved quantities, which are global objects concerning the whole system, for its description we must switch over from the local YBE (2.1) to some global relations. For this purpose therefore, we define a global object known as the monodromy matrix, by matrixmultiplying the Lax operators: $T_{a}(\lambda)=\prod_{j=1}^{N} L_{a j}(\lambda)$, which yields again a $p \times p$ matrix with operator elements $T_{\alpha \beta}$. Multiplying the local relations (2.1) successively for all sites $j$ we arrive at the global YBE exhibiting the same form:

$$
R_{a b}(\lambda-\mu) T_{a}(\lambda) T_{b}(\mu)=T_{b}(\mu) T_{a}(\lambda) R_{a b}(\lambda-\mu)
$$

Note that the construction of this global YBE is possible only due to the ultralocality property, when the Lax operators at different sites can be treated almost like commuting classical objects and can be dragged through one another and multiplied to yield (2.3). This algebraic invariance of the tensor product reflects the deep Hopf algebra structure underlying all integrable systems. Taking now the trace from both sides of relation (2.3), the $R$-matrices get canceled, since they can be rotated cyclically under the trace and one gets finally for the transfer matrix $\tau(\lambda)=\operatorname{tr}(T(\lambda))$ the crucial relation $[\tau(\lambda), \tau(\mu)]=0$. Defining the conserved quantities as the expansion coefficients of

$$
\ln \tau(\lambda)=\sum_{n} C_{n} \lambda^{n}
$$

the commutativity of the set $\left\{C_{n}\right\}$ for different values of $n$ : $\left[C_{n}, C_{m}\right]=0$, follows immediately and that establishes the complete quantum integrability of the system. 


\subsection{Hamiltonian construction}

Though the starting point in a Quantum integrable system is an abstract Lax operator, it can systematically generate the explicit forms of all conserved operators of physical interest including the Hamiltonian of the system. This follows from the fact that the Lax operators through successive multiplication leads to the monodromy matrix and the trace of it gives the transfer matrix, which in turn using (2.4) generates the conserved quantities $C_{n}=\left.\frac{1}{n !} \frac{\partial}{\partial \lambda} \ln \tau(\lambda)\right|_{\lambda=0}$. For example, if the Hamiltonian of the model is defined as $H \equiv C_{1}=\tau^{\prime}(0) \tau^{-1}(0)$ and the Lax operator satisfies an important criterion called regularity condition: $L_{a j}(0)=P_{a j}$, linking it with the permutation operator $P_{a j}$, then the Hamiltonian may be expressed directly through the Lax operator as

$$
H=\sum_{j} H_{j j+1}, \quad H_{j j+1}=L_{j j+1}^{\prime}(0) L_{j j+1}^{-1}(0)=L_{j j+1}^{\prime}(0) P_{j j+1} .
$$

Note that due to the regularity condition of the Lax operator one can use the properties of the permutation operator: $P_{a j} L_{a j+k}=L_{j j+k} P_{a j}$ and $P_{a j}^{2}=\operatorname{tr}_{a} P_{a j}=I$ to derive Hamiltonian (2.5), exhibiting nearest neighbour interactions. Fortunately, fundamental integrable models in condensed matter physics, e.g. spin models, Hubbard model, $t-J$ model etc. fall into the category of ultralocal as well as regular model and their Hamiltonians are with nearest neighbour interactions described by $C_{1}$. The theory of nonultralocal integrable models is still in the developmental stage [19] and will not be considered here.

\subsection{Algebraic Bethe ansatz}

As we have stated above, while the CBA is concerned with the exact solution of the EVP for the Hamiltonian $H$, the ABA aims to do so for all conserved operators simultaneously. This is achieved by solving the EVP for the transfer matrix, expressed through the diagonal elements of the monodromy matrix as $\tau(\lambda)=\sum_{\alpha} T_{\alpha \alpha}(\lambda)$. The off-diagonal elements of the monodromy matrix $T_{\alpha \beta}\left(\lambda_{i}\right)$ with $\alpha<\beta$, on the other hand correspond usually to the pseudoparticle creation operators and their successive actions on the pseudovacuum $\mid 0>$ generate the $n$-particle eigenstates $\mid n>$. The states $\mid n>$ and consequently the corresponding eigenvalues of $\tau(\lambda)$ de-

pends on $p-1$ different sets of spectral parameters $\left\{\lambda_{i_{r}}^{(r-1)}\right\}, r=1, \ldots, p-1$ also known as the rapidity variables, such that the total number of excitations matches with the pseudoparticle number: $\sum i_{r}=n$. Therefore for calculating the related eigenvalue problem, one has to examine the actions of the diagonal elements $T_{\alpha \alpha}(\lambda) \mid n>$, for which one needs the explicit commutation relations between the diagonal and the off-diagonal elements of $T(\lambda)$. This knowledge is required for dragging the former type of operators through the later type until they hit the pseudovacuum state $\mid 0>$. The resulting problem $T_{\alpha \alpha}(\lambda)\left|0>=a_{\alpha}(\lambda)\right| 0>$ can be readily solved, 
since $a_{\alpha}(\lambda)$ is obtained from the trivial action of the Lax operators on the pseudovacuum state. Demanding $\mid n>$ to be the true eigenstate of $\tau(\lambda)$, one gets on the other hand some important extra conditions, known as the Bethe equations, for determining the rapidity variables $\left\{\lambda_{i_{r}}^{(r-1)}\right\}$. The exact procedure of ABA depends naturally on the concrete models. Recalling $p-1$ as the rank of the underlying algebra, we see that for systems with $p=2$, e.g. for the spin- $\frac{1}{2}$ chain there is only a single set of rapidities $\left\{\lambda_{i}\right\}$, while for $m$-chain spin models, one gets $m$ sets of rapidities $\left\{\lambda_{i_{r}}^{(r-1)}\right\}, r=1, \ldots, m$. Similarly, the Hubbard and the supersymmetric $t-J$ models

are described by two sets of rapidities $\left\{\lambda_{j}^{(0)}, \lambda_{\alpha}^{(1)}\right\}$. Note however that the underlying symmetry algebra of the Hubbard model is $s u(2) \times s u(2)$, resulting a $4 \times 4$ Lax operator, while that for the SUSY $t-J$ model is $g l(1,2)$ with its Lax operator being a $3 \times 3$ matrix. For models with $p>2$ the ABA steps are recursively repeated giving the nested Bethe ansatz. We shall give some details on the twisted Hubbard and the $t-J$ models in sect. 6 for elaborating this process.

\section{$3 \quad$ Symmetries of YBE and Generating transformations}

For identifying a procedure for generating integrable multi-chain electron models with correlated hopping and spin models with interchain interactions we intend to find certain symmetries of the YBE, which would yield under particular transformation Lax operators and $R$-matrices as new solutions of the YBE. Therefore the idea is to start with the $L, R$ matrices of known integrable systems and derive the transformed ones satisfying again the YBE and therefore representing new integrable systems. From these transformed Lax operators one can derive now the Hamiltonians of the desired integrable models with correlated hopping.

We look for the set of transformations given by

$$
R_{a b}(\lambda, \mu) \rightarrow \tilde{R}_{a b}(\lambda, \mu)=F_{a b} R_{a b}(\lambda, \mu) F_{b a}^{-1} ; \quad \tilde{L}_{a j}(\lambda)=F_{a j} L_{a j}(\lambda) F_{j a}^{-1}
$$

and demand that the YBE (2.1) should remain valid under such a transformation. In what follows we will frequently denote $L_{a j}$ by $R_{a j}$ for convenience. It can be checked easily through simple algebra that, (3.1) maps a solution of the YBE into another one, only if the following set of relations holds.

$$
R_{a b} F_{a j} F_{b j}=F_{b j} F_{a j} R_{a b}, \quad R_{a j} F_{b j} F_{b a}=F_{b a} F_{b j} R_{a j}, \quad R_{b j} F_{b a} F_{j a}=F_{b a} F_{j a} R_{b j},
$$

along with the conditions that $F_{a b}$ must itself be a solution of the YBE (2.2):

$$
F_{a b} F_{a c} F_{b c}=F_{b c} F_{a c} F_{a b}
$$

In our constructions we will explicitly find the transforming operator $F$ by assuming certain symmetries. For example, supposing the symmetric condition $F_{b a}=F_{a b} \equiv S_{a b}$, transformation 
(3.1) looks like a gauge transformation

$$
\tilde{R}_{a b}(\lambda, \mu)=S_{a b} R_{a b}(\lambda, \mu) S_{a b}^{-1} ; \quad \tilde{L}_{a j}(\lambda)=S_{a j} L_{a j}(\lambda) S_{a j}^{-1},
$$

while with anti-symmetry $F_{b a}^{-1}=F_{a b} \equiv T_{a b}$, which is known as the twisting transformations [20] we get

$$
\tilde{R}_{a b}(\lambda, \mu)=T_{a b} R_{a b}(\lambda, \mu) T_{a b} ; \quad \tilde{L}_{a j}(\lambda)=T_{a j} L_{a j}(\lambda) T_{a j}
$$

For model construction we consider transformations with such symmetries only and satisfy trivially the essential condition (3.3) by choosing the transforming operators $F$ as mutually commuting. Notice that under these conditions all relations in (3.2) can be reduced for both the gauge and the twisting transformations to a single convenient form

$$
R_{a b} F_{a c} F_{b c}=F_{b c} F_{a c} R_{a b}
$$

where the indices $a, b, j$ are treated in equal footing with $R_{a j}$ to be understood as $L_{a} j$.

There exists another type of gauge transformation, where the symmetric operators are given in the factorised form: $S_{a b} \equiv G_{a b}=g_{a} g_{b}$. It can be shown easily that, in such cases no extra condition is imposed for the validity of the transformed solutions and the operators $g_{a}$ can be arbitrary invertible matrix. To demonstrate this we may focus on the lhs of the YBE (2.1) and insert the transformed solutions (3.1) with the transforming operators in the factorised form to get

$$
\begin{aligned}
\mathrm{lhs} & =g_{a} g_{b} R_{a b}(\lambda, \mu) g_{a}^{-1} g_{b}^{-1} g_{a} g_{j} L_{a j}(\lambda) g_{a}^{-1} g_{j}^{-1} g_{b} g_{j} L_{b j}(\mu) g_{b}^{-1} g_{j}^{-1} \\
& =g_{a} g_{b} g_{j}\left(R_{a b}(\lambda, \mu) L_{a j}(\lambda) L_{b j}(\mu)\right) g_{a}^{-1} g_{b}^{-1} g_{j}^{-1}
\end{aligned}
$$

Similar calculations are repeated for the rhs and since the old $R, L$ solutions satisfy the YBE, it holds naturally for the transformed solutions. Note that in dragging the factors involving $g_{a}$ 's out of the original lhs of YBE as done in (3.7), we have used only the mutual cancellation of these factors as well as trivial commutativity of the operators acting on different spaces. For such transformations as evident from (3.7) the validity of the YBE condition (3.3) is also not required. Therefore the choice of the transforming operators $g_{a}$ can be completely arbitrary. We show below that using such freedom we can construct integrable models which can go beyond the class of models classified in [15]. However confining only to mutually commuting twisting and gauge transformations, as mentioned above, we can derive an integrable subclass of the solvable models considered in [15]. We emphasise again that all the models we generate here belong to the quantum integrable systems and therefore are much richer than the models, which allow only the solution of the Hamiltonian eigenvalue problem through CBA. 


\subsection{Transformed Hamiltonian}

Recall that (2.5) links Hamiltonian of a model directly to its Lax operator, if the regularity condition is fulfilled. We notice further that the regularity condition is preserved under transformations (3.1), since it gives $\tilde{L}_{a j}(0)=F_{a j} L_{a j}(0) F_{j a}^{-1}=F_{a j} P_{a j} F_{j a}^{-1}=F_{a j} F_{a j}^{-1} P_{a j}=P_{a j}$. Therefore one gets a precise way for obtaining new transformed Hamiltonians explicitly from the transformed Lax operators (3.1) as

$$
\begin{aligned}
H & =\sum_{j} \tilde{L}_{j j+1}^{\prime}(0) \tilde{L}_{j j+1}^{-1}(0), \\
& =\sum_{j}\left(F_{j j+1} L_{j j+1}^{\prime}(0) F_{j+1 j}^{-1}\right)\left(F_{j+1 j} L_{j j+1}^{-1}(0) F_{j j+1}^{-1}\right), \\
& =\sum_{j} F_{j j+1}\left(H_{j j+1}\right) F_{j j+1}^{-1},
\end{aligned}
$$

where $H_{j j+1}$ corresponds to the original model as defined in (2.5). Therefore if one starts from known integrable models like $X X Z$ or Hubbard model, with their Lax operators satisfying both ultralocality and regularity conditions, then through (3.1) one can derive the transformed Lax operator with the same essential properties and in turn can generate through (3.8) the Hamiltonian with new interactions introduced by the operator $F_{j j+1}$.

\section{Model construction}

For constructing new integrable models following the above scheme we restrict ourselves only to the symmetric (3.4) and antisymmetric (3.5) transformations and build the transforming operators out of the Cartan generators $H_{(\alpha)}, \alpha=1, \ldots, r, r=p-1$ being the rank of the underlying algebra. Due to the mutual commutativity of $H_{j}$ the transforming operators would automatically satisfy the YBE (3.3) and therefore the only condition that remains to be satisfied

for our transformations $S_{a b}$ and $T_{a b}$ is (3.6). It can be shown that such operators in the general case may be constructed in the exponential form 20]

$$
\mathrm{G}_{a j}=e^{i \sum_{\alpha \beta} \theta_{\alpha \beta}\left(H_{a(\alpha)} H_{j(\beta)}\right)},
$$

with symmetric or antisymmetric properties on the parameters: $\theta_{\beta \alpha}= \pm \theta_{\alpha \beta}$. In (4.1) $\alpha=0$ may also be included in the sum by considering $H_{0} \equiv 1$. Note that the rank $r$ of the associated algebra depends on the concrete models and determines also the matrix dimension of the Lax operator together with the chosen representation. However since we are interested here in a particular class of models with relevance in condensed matter physics, e.g. spin chains, Hubbard model, t-J model etc. we would prove the validity of (4.1) in such particular cases only. 


\subsection{Correlated electron and spin ladder models}

We consider first the Hubbard like correlated electron models involving operators $c_{j(\alpha)}^{\dagger}, c_{j(\alpha)}, n_{j(\alpha)}$ with spin components $\alpha= \pm$ and $X X Z$ or $X X X$ type two-chain or the so called ladder models involving two independent spin- $\frac{1}{2}$ operators $\vec{\sigma}, \vec{\tau}$. Subsequently, we will look into their multichain generalisations as well as transformed $t-J$ and some other new spin models. Note that since through Jordan-Wigner transformation the spin operators can be mapped into fermions and vice versa, the Lax operators in case of spin models can be obtained analogous to those for the fermionic models, which we consider here in details. Therefore in conformity with (4.1) we propose the symmetric gauge transformations as

$$
S_{a j}(s)=e^{i s\left(n_{a(-)} n_{j(+)}+n_{a(+)} n_{j(-)}\right)} .
$$

while the antisymmetric twisting transformation in the form

$$
T_{a j}\left(\theta, \gamma_{( \pm)}\right)=e^{i\left[\theta\left(n_{a(-)} n_{j(+)}-n_{a(+)} n_{j(-)}\right)+\gamma_{(+)}\left(n_{a(+)}-n_{j(+)}\right)+\gamma_{(-)}\left(n_{a(-)}-n_{j(-)}\right)\right.}
$$

As we have stated above, we have to check only the validity of (3.6) for both these operators to confirm their usefulness for generating new integrable systems. In all our calculations we need only to use the following basic commutation relations for fermions or spin operators like

$$
\begin{gathered}
f\left(n_{a(\beta)}\right) c_{j(\alpha)}^{\dagger}=\delta_{a j} \delta_{\alpha \beta} c_{j(\alpha)}^{\dagger} f\left(n_{a(\beta)}+1\right), \quad f\left(n_{a(\beta)}\right) c_{j(\alpha)}=\delta_{a j} \delta_{\alpha \beta} c_{j(\alpha)} f\left(n_{a(\beta)}-1\right) \\
{\left[\sigma^{ \pm}, \sigma^{3}\right]=\mp 2 \sigma^{ \pm}, \quad\left[\sigma^{+}, \sigma^{-}\right]=\sigma^{3}, \quad\left(\sigma^{ \pm}\right)^{2}=0}
\end{gathered}
$$

etc. We note first that the $R_{a b}$-matrix or the Lax operator of the Hubbard model (similarly those for the $X X Z$ or $X X X$ spin models) contains strings of operators in the quadratic

form $c_{a( \pm)}^{\dagger} c_{b( \pm)}$, which are the only parts not commuting with the operators $n_{a( \pm)}$ forming the above transformations. However it is evident that, any function of the total number operator $n_{a( \pm)}+n_{b( \pm)}$ commutes with this quadratic form and hence with the $R_{a b}$, while the operator $n_{c( \pm)}$ commutes trivially with it. We note now that the transforming matrices in (3.6) induce operators exactly in such combinations: $F_{a c} F_{b c} \sim e^{i c o n s t .\left(\left(n_{a(-)}+n_{b(-)}\right) n_{c(+)} \pm\left(n_{a(+)}+n_{b(+)}\right) n_{c(-)}\right)}$, for (4.2) and (4.3), respectively, which based on our above arguments proves the validity of (3.6) and therefore the validity of (3.1) for both (4.2) and (4.2). We may consider also an additional transformation in the factorised form choosing

$$
G_{a j}(g)=g_{a} g_{j}, \quad g_{a}(g)=e^{i g\left(n_{a(-)} n_{a(+)}\right)} .
$$

Since (4.2), (4.3) and (4.6) can be applied independently, we may consider their combined effect by their successive application:

$$
F_{a j}\left(\theta, \gamma_{( \pm)}, s, g\right)=S_{a j}(s) T_{a j}\left(\theta, \gamma_{( \pm)}\right) G_{a j}(g), \quad \tilde{F}_{a j}\left(\theta, \gamma_{( \pm)}, s, g\right)=S_{a j}^{-1}(s) T_{a j}\left(\theta, \gamma_{( \pm)}\right)\left(G_{a j}\right)^{-1}(g)
$$


for constructing transformation through (3.1) as

$$
\tilde{R}_{a j}\left(\theta, \gamma_{( \pm)}, s, g, \lambda\right)=F_{a j}\left(\theta, \gamma_{( \pm)}, s, g\right) R_{a j}^{(H u b)}(\lambda) \tilde{F}_{a j}\left(\theta, \gamma_{( \pm)}, s, g\right)
$$

Here the transformed $\tilde{R}$ in (4.8) stands for both the changed Lax operator and the $R$-matrix and naturally satisfies the YBE representing a new quantum integrable system with $N$ number of independent conserved operators. These systems would give a hierarchy of extended Hubbard models with correlated hopping or through Jordan-Wigner mapping from fermion to spin

operator: $n_{a( \pm)} \rightarrow \frac{1}{2}\left(1+\sigma_{a( \pm)}^{3}\right)$ and $c_{j( \pm)} \rightarrow \sigma_{j( \pm)}^{+}, c_{j( \pm)}^{\dagger} \rightarrow \sigma_{j( \pm)}^{-}$, a hierarchy of spin ladder models with interchain interactions. We have used here the notation $\vec{\sigma}_{(-)}$in place of $\vec{\tau}$ for convenience.

The Hamiltonian of such transformed Hubbard systems would be given by (3.8) with the use of the transformation (4.7) as

$$
\begin{aligned}
\tilde{H} & =\sum_{j} F_{j j+1}\left(\theta, \gamma_{( \pm)}, s, g\right) H_{j j+1}^{(H u b)} F_{j j+1}^{-1}\left(\theta, \gamma_{( \pm)}, s, g\right) \\
& =-\sum_{j} c_{j+1(+)}^{\dagger} c_{j(+)} h_{j j+1}^{(+)}+c_{j+1(-)}^{\dagger} c_{j(-)} h_{j j+1}^{(-)}+U n_{j(+)} n_{j(-)}+\text { h.c }
\end{aligned}
$$

exhibiting explicit correlated hopping

$$
h_{j j+1}^{( \pm)}=e^{i\left(-2 \gamma( \pm)+( \pm \theta-s+g) n_{j+1(\mp)}+( \pm \theta+s-g) n_{j(\mp)}\right)} .
$$

Its different parameters keep track of the independent transformations (4.2), (4.3), (4.6) and therefore one can study each or any combinations of them by switching off the others. In this way, as we see below, one can identify different known models showing also the equivalence between them.

\subsection{Connection with general setting and equivalence}

We can now link our model to the correlated hopping electron models classified in [15]. This comparison reveals that our model described by the Hamiltonian (4.9) with (4.10) constitutes an important integrable subclass of the solvable models of [15], defined by a particular choice of their coefficients $\alpha_{j m}(\sigma)$ and $\gamma_{j}(\sigma)$ appearing in the correlated hopping terms as

$$
\alpha_{j m}(\sigma)=\delta_{j m}(\sigma \theta+s-g)+\delta_{j+1 m}(\sigma \theta-(s-g)), \quad \gamma_{j}(\sigma)=-2 \gamma_{(\sigma)} .
$$

where $\sigma= \pm$. Therefore the global unitary transformation defined in 15

$$
U=\exp \left(i \sum\left(\xi_{j, k}^{\alpha, \beta} n_{j(\alpha)} n_{k(\beta)}+\zeta_{j, \alpha} n_{j(\alpha)}\right)\right)
$$


that introduces correlated hopping in the Hamiltonian can also be identified for our case by equating the coefficients as

$$
\xi_{j, j}^{\sigma,-\sigma}=\xi_{j+1, j+1}^{\sigma,-\sigma}=-\frac{1}{2}(s-g), \quad \xi_{j, j+1}^{\sigma,-\sigma}=-\xi_{j+1, j}^{\sigma,-\sigma}=\frac{1}{2} \sigma \theta
$$

with the rest of the coefficients in (4.12) including all $\xi_{j, k}^{\sigma, \sigma}$ being zero. As a consequence all coefficients $A_{j, k}(\sigma)=2\left(\xi_{j, k}^{\sigma, \sigma}-\xi_{j+1, k}^{\sigma, \sigma}\right), k \neq(j, j+1)$ appearing in the models of 15 also vanish. Moreover it is significant to note that the class of quantum integrable models we construct correspond to the translational invariant subclass of the solvable models [15, as is evident from (4.11), since we have here $\alpha_{j m}(\sigma)=\alpha_{j-m}(\sigma)$ with its only nontrivial coefficients $\alpha_{0}( \pm)=$ $\pm \theta+s-g$ and $\alpha_{-1}(\mp)= \pm \theta-(s-g)$, satisfying the necessary condition $\alpha_{0}( \pm)+\alpha_{-1}(\mp)=0$. Weather this linkage between the quantum integrability and the translational invariance has any deeper meaning is yet to be explored. On the other hand we recall that the models introduced by Schulz-Shastry [13] also exhibits translational invariance, since their coefficients correspond to $\alpha_{j m}( \pm)=\mp \alpha_{j-m}$ with $A_{j, k}(\sigma)=\gamma_{j( \pm)}=0$. Comparing with (4.11) we may notice that, the Schulz-Shastry model, in spite of its translational invariance, does not in general correspond to our quantum integrable case. However, we will see below that a further restricted model, known as the minimal Schulz-Shastry model, given by the particular choice $\alpha_{0}=\alpha_{-1} \neq 0$ with all other $\alpha_{k}=0$ does agree with a particular case of the present construction, showing that the minimal Schulz-Shastry model belongs to a quantum integrable class. Returning again to the present case, we observe that just by restricting to the choice (4.11) and (4.13), we can use all the formulas derived in [15] for our model and thus can calculate the set of boundary phases and other relevant objects for the present case. Moreover, we can find that the necessary condition for the CBA solvability of such models prescribed in [15], holds also for our model, since for the choice (4.11) it is easy to check that the compatibility condition: $\alpha_{j m}(\sigma)+\alpha_{m j+1}(-\sigma)-\alpha_{j m+1}(\sigma)-\alpha_{m j}(-\sigma)=0$ with $m=j, j+1$ only, holds true.

Therefore we conclude that, while all Amico et al model [15] are only CBA solvable, their particular subclass defined by (4.11) and (4.13) and generated by our construction not only becomes CBA solvable but also represents an exact quantum integrable system. This integrable subclass may be given by the Hamiltonian (4.9) with (4.10) together with all its particular cases and is associated with explicit Lax operator and $R$-matrix solutions (4.8). Consequently, these models allow a hierarchy of mutually commuting conserved operators having increasingly further neighbour interactions, e.g. nearest, next nearest, second next nearest neighbours etc., as well as more and more nonlinear interacting terms in their successive Hamiltonians. Moreover, as we have mentioned already, while CBA aims to solve the eigenvalue problem for the Hamiltonian of a single model, quantum integrable systems permit exact eigenvalue solution for all its $N$-number of conserved operators, simultaneously through ABA. We will demonstrate briefly this ABA application to our integrable model (4.9), (4.10) in sect. 6. 
We stress again that one can generate various extensions to the Hubbard model from (4.9) by adjusting the independent parameters $\gamma_{( \pm)}, \theta, s, g$ in (4.10), all of which would be quantum integrable systems allowing application of ABA, as well as solvable through CBA models. Their Lax operators and $R$-matrices would naturally be related to each other by gauge or twisting transformations giving a direct way of establishing explicit relationship between the models with different types of hopping interactions at their Lax operator level. This throws a new light on the question of equivalence of the models, which created significant controversy in recent years [18, 17, 15]. For example, if we restrict only to the twisting transformation by choosing $\theta \neq 0$, while switching off the other parameters: $g=s=\gamma_{( \pm)}=0$, we get an integrable model with the Lax operator

$$
\tilde{R}_{a j}(\theta, \lambda)=T_{a j}(\theta, 0) R_{a j}^{(H u b)}(\lambda) T_{a j}(\theta, 0)
$$

with $T_{a j}(\theta, 0)=e^{i\left[\theta\left(n_{a(-)} n_{j(+)}-n_{a(+)} n_{j(-)}\right)\right.}$ as in (4.3) and the corresponding Hamiltonian will be given as (4.9) with its hopping interaction reduced accordingly from (4.10) as

$$
\begin{aligned}
\tilde{H} & =-\sum_{j}\left(c_{j+1(+)}^{\dagger} c_{j(+)} e^{i \theta\left(n_{j(-)}+n_{j+1(-)}\right)}+c_{j+1(-)}^{\dagger} c_{j(-)} e^{-i \theta\left(n_{j(+)}+n_{j+1(+)}\right)}\right. \\
& \left.+U n_{j(+)} n_{j(-)}+\text { h.c. }\right)
\end{aligned}
$$

We observe that (4.14) coincides with the $L$-operator found in [14, while (4.15) recovers the Hamiltonian of the Kundu model [14], which also tallies interestingly with the minimal SchulzShastry model [13, 18]. Clearly, from (4.15) one derives $\alpha_{0}=\alpha_{-1}=\theta$ as the only nonvanishing coefficients in its correlated hopping term, which defines the minimal Schulz-Shastry model, as mentioned above. We therefore conclude that the minimal Schulz-Shastry model $H^{(\min -S S)}$, given by the Hamiltonian (4.15) and identical to the model of [14] represents a quantum integrable system with its Lax operator and the $R$-matrix $R_{a j}^{(\min -S S)}(\theta, \lambda)$ given by (4.14).

Let us consider now a combination of the gauge and the twisting transformation by adjusting the corresponding parameters as $s=\theta$, while switching off the others by choosing $g=\gamma_{( \pm)}=0$. The resulting integrable system will have the Lax operator and the $R$-matrix (4.8) with the transforming operators $F_{a j}(\theta)=S_{a j}(\theta) T_{a j}(\theta, 0)=e^{\left.2 i \theta\left(n_{a(-)} n_{j(+)}\right)\right)}, \quad \tilde{F}_{a j}(\theta)=S_{a j}^{-1}(\theta) T_{a j}(\theta, 0)=$ $e^{-2 i \theta\left(n_{a(+)} n_{j(-)}\right)}$, expressed in the form

$$
\tilde{R}_{a j}(\theta, \lambda)=e^{\left.2 i \theta\left(n_{a(-)} n_{j(+)}\right)\right)} R_{a j}^{(H u b)}(\lambda) e^{-2 i \theta\left(n_{a(+)} n_{j(-)}\right)}
$$

for the extended Hubbard model and similarly

$$
\tilde{R}_{a j}(\theta, \lambda)=e^{2 i \theta\left(\tau_{a}^{3} \sigma_{j}^{3}\right)}\left(R_{a j}^{(x x z) \sigma}(\lambda) R_{a j}^{(x x z) \tau}(\lambda)\right) e^{-2 i \theta\left(\sigma_{a}^{3} \tau_{j}^{3}\right)}
$$

for the XXZ ladder model. It is exciting to recognise that Lax operator (4.16) (or (4.17)) is exactly same as that for the extended Hubbard model $R_{a j}^{(Z)}(\theta, \lambda)$ constructed by Zvyagin et al 
[11, 12] for their single chain case. Comparing (4.16) and (4.14) we observe therefore that these Lax operators are related only by an unitary transformation and consequently we come to the important conclusion that the Zvyagin et al model is a quantum integrable model and is gauge equivalent to the minimal Schulz-Shastry model at the Lax operator level, i.e. we have

$$
R_{a j}^{(Z)}(\theta, \lambda)=S_{a j}(\theta) R_{a j}^{(\min -S S)}(\theta, \lambda) S_{a j}^{-1}(\theta), \quad S_{a j}(\theta)=e^{i\left[\theta\left(n_{a(-)} n_{j(+)}+n_{a(+)} n_{j(-)}\right)\right.}
$$

Moreover since the Hamiltonian can be determined uniquely from the Lax operator as shown in (2.5), the equivalence between the above two models extends also to their Hamiltonian level. However, this equivalence remained obscured [18] possibly due to the fact that in spite of the correct form of the Lax operator in [11, 12], the corresponding Hamiltonian as presented in the same papers [11, 12] involves some error [21]. As a consequence this form of the Hamiltonian appears to be even nonsolvable by CBA, as observed in [15], whereas being associated with solutions of YBE it should certainly be an exact quantum integrable model! Our approach resolves this puzzle by deriving the correct form of the Hamiltonian from (3.8) using the Lax operator $R_{a j}^{(Z)}(\theta, \lambda)$ of [11, 12], which coincides with (4.16). Therefore one concludes that the correct Zvyagin et al model $H^{(Z)}$ should be given by (4.9) through a reduction of the parameters $g=\gamma_{( \pm)}=0, s=\theta$ in the hopping interaction (4.10) yielding

$$
\begin{aligned}
\tilde{H} & =\sum_{j} e^{\left.2 i \theta\left(n_{j(-)} n_{j+1(+)}\right)\right)} H_{j j+1}^{(H u b)} e^{\left.-2 i \theta\left(n_{j(-)} n_{j+1(+)}\right)\right)} \\
& =-\sum_{j} c_{j+1(+)}^{\dagger} c_{j(+)} e^{2 i \theta n_{j(-)}}+c_{j+1(-)}^{\dagger} c_{j(-)} e^{-2 i \theta n_{j+1(+)}}+U n_{j(+)} n_{j(-)}+\text { h.c. }
\end{aligned}
$$

It follows therefore from the above arguments that, the proper Hamiltonian of the Zvyagin et al model, which should be given by (4.19) is related through unitary transformation to that of the minimal Schulz-Shastry model, which coincides with (4.15). That is one should have $H^{(Z)}=U_{1} H^{(\min -S S)} U_{1}^{-1}$, where the global unitary operator $U_{1}$ can be constructed easily from (4.12) and (4.13) as $U_{1}=\exp \left(-i \theta \sum_{j}\left(n_{j(+)} n_{j(-)}\right)\right)$, which establishes the gauge equivalence between these two models also at the Hamiltonian level. An alternative choice of $s=-\theta$, leads to a complementary model with Hamiltonian (4.9) and correlated hopping $h_{j j+1}^{(+)}=e^{2 i \theta n_{j+1(-)}}$ and $h_{j j+1}^{(-)}=e^{-2 i \theta n_{j(+)}}$, which again is gauge equivalent to the minimal Schulz-Shastry model and can be taken also as an alternative but correct form of the Zvyagin et al model. Since both of the above models are obtained as particular cases of (4.9), they must be CBA solvable as well as quantum integrable systems. Based on our explicit results derived below, we may draw another important conclusion that, contrary to the Hamiltonian of the models the Bethe ansatz results are insensitive to the gauge transformations $\mathrm{S}(4.2$ ) and $\mathrm{G}$ (4.6) and depend only on the twisting transformation $\mathrm{T}(4.3)$. Therefore, since at the Lax operator level the Zvyagin et al model is S-gauge related to the minimal Schulz-Shastry model, which again is identical 
to the Kundu model, for all these three models the scattering matrix and the Bethe equations related to their eigenvalue problem turn out to be identical. This is explicit from the identical Bethe ansatz equations presented in respective works [11, 12, 13, 14] and possibly this is the reason why the final Bethe ansatz results in [11, 12] turn out to be correct in spite of their incorrect form of the Hamiltonian.

\subsection{Multi-chain generalization}

In the above models we have taken $\alpha= \pm$ for denoting the $\uparrow, \downarrow$ components of a $s=\frac{1}{2}$ single chain electron or the two independent spin- $\frac{1}{2}$ operators in a spin ladder model. For generalizing these models to M-chains we have to consider the set of fermionic operators $\left\{c_{j(\alpha)}^{\dagger}, c_{j(\alpha)}, n_{j(\alpha)}\right\}$ or spin operators $\vec{\sigma}_{(\alpha)}$ extending the range of $\alpha$ to $\alpha=1, \ldots, M$. Note that we can incorporate easily multi-chain electron models with spin- $\frac{1}{2}$ by redefining the indices through a break up of $\alpha=(\gamma, \pm), \gamma=1, \ldots, \frac{M}{2}$, resulting $\frac{M}{2}$ number of electron chains. Since the rest of the arguments go parallelly, we will not distinguish spin-less fermions from fermions with spins in what follows, for convenience. Moreover since the idea of construction described above for the single chain models allows simple generalization to the multi-chain case, we will give only brief account of such constructions skipping the details and stressing only the differences. Firstly for generalising the above gauge and twisting operators (4.2), (4.3) and (4.6) to the $M$-chain models, we have to use now more general transformations (4.1) to get the transforming operators as

$$
\begin{gathered}
S_{a j}^{(M)}(\hat{s})=e^{i \sum_{\alpha<\beta} s_{\alpha \beta}\left(n_{a(\alpha)} n_{j(\beta)}+n_{a(\beta)} n_{j(\alpha)}\right)} \\
T_{a j}^{(M)}(\hat{\theta}, \vec{\gamma}, \hat{\gamma})=e^{i\left(\sum_{\alpha<\beta} \theta_{\alpha \beta}\left(n_{a(\alpha)} n_{j(\beta)}-n_{a(\beta)} n_{j(\alpha)}\right)+\sum_{\alpha} \gamma_{(\alpha)}\left(n_{a(\alpha)}-n_{j(\alpha)}\right)\right)}
\end{gathered}
$$

and

$$
G_{a j}^{(M)}(\hat{g})=g_{a}^{(M)} g_{j}^{(M)}, \quad g_{a}^{(M)}(\hat{g})=e^{i \sum_{\alpha<\beta} g_{\alpha \beta}\left(n_{a(\alpha)} n_{a(\beta)}\right)},
$$

respectively, considering the parameters $\hat{s}, \hat{\theta}, \hat{g}$ to be symmetric under interchange of indices, i.e. $\theta_{\alpha \beta}=\theta_{\beta \alpha}$ etc. The Lax operator and the $R$-matrix of the new quantum integrable multi-chain model can be constructed generalizing (4.8) as

$$
\tilde{R}_{a j}^{(M)}(\hat{\theta}, \vec{\gamma}, \hat{s}, \hat{g}, \lambda)=F_{a j}^{(M)}(\hat{\theta}, \vec{\gamma}, \hat{s}, \hat{g}) L_{a j}^{(M) H u b}(\lambda) \tilde{F}_{a j}^{(M)}(\hat{\theta}, \vec{\gamma}, \hat{s}, \hat{g})
$$

where the transforming operators (4.7) are generalised by using the combinations of multi-chain twisting and gauge transformation (4.20)-(4.22) as

$$
F_{a j}^{(M)}(\hat{\theta}, \vec{\gamma}, \hat{s}, \hat{g})=T_{a j}^{(M)}(\hat{\theta}, \vec{\gamma}) S_{a j}^{(M)}(\hat{s}) G_{a j}^{(M)}(\hat{g}), \quad \tilde{F}_{a j}^{(M)}(\hat{\theta}, \vec{\gamma}, \hat{s}, \hat{g})=T_{a j}^{(M)}(\hat{\theta}, \vec{\gamma})\left(S_{a j}^{(M)}(\hat{s}) G_{a j}^{(M)}(\hat{g})\right)^{-1}
$$


The set of conserved operators, the Hamiltonian being first in this set, can be constructed in explicit form through a straightforward generalization of (3.8) for the $M$-chain case by using the transforming operators from (4.24). Since the transformations considered here do not affect the interacting term in the Hamiltonian involving Cartan generators, we present only its transformed hopping or the $X Y$ terms given as

$$
\begin{aligned}
\tilde{H}^{(M)} & =\sum_{j}^{N} F_{j j+1}^{(M)}(\hat{\theta}, \vec{\gamma}, \hat{s}, \hat{g})\left(H_{j j+1}^{(M) X Y}\right)\left(F_{j j+1}^{(M)}(\hat{\theta}, \vec{\gamma}, \hat{s}, \hat{g})\right)^{-1} \\
& =-\sum_{j, \alpha}^{N, M} c_{j+1(\alpha)}^{\dagger} c_{j(\alpha)} h_{j j+1}^{(\alpha)}+\text { h.c }
\end{aligned}
$$

with correlated hopping involving all other chains:

$$
h_{j j+1}^{(\alpha)}=e^{i\left(-2 \gamma(\alpha)+\sum_{\beta \neq \alpha}\left(\operatorname{sgn}(\alpha-\beta) \theta_{\alpha \beta}\left(n_{j+1(\beta)}+n_{j(\beta)}\right)+\left(s_{\alpha \beta}-g_{\alpha \beta}\right)\left(n_{j(\beta)}-n_{j+1(\beta)}\right)\right)\right.} .
$$

Comparing with the classification of [15] we find that, our Hamiltonian (4.25) is obtained for the particular choice of their parameters

$A_{j m}^{\alpha, \beta}=\delta_{j m}\left(\operatorname{sgn}(\alpha-\beta) \theta_{\alpha \beta}+s_{\alpha \beta}-g_{\alpha \beta}\right)+\delta_{j+1 m}\left(\operatorname{sgn}(\alpha-\beta) \theta_{\alpha \beta}-\left(s_{\alpha \beta}-g_{\alpha \beta}\right)\right), \quad \gamma_{j}(\alpha)=-2 \gamma(\alpha)$,

with $\beta \neq \alpha$, which may be considered as the $M$-chain generalization of the relation (4.11). Consequently the only nontrivial coefficients appearing in the corresponding global unitary transformation (4.12) that introduces correlated hopping in the Hamiltonian would be given by

$$
\xi_{j, j}^{\alpha, \beta}=\xi_{j+1, j+1}^{\alpha, \beta}=-\frac{1}{2}\left(s_{\alpha, \beta}-g_{\alpha, \beta}\right), \quad \xi_{j, j+1}^{\alpha, \beta}=-\xi_{j+1, j}^{\alpha, \beta}=\frac{1}{2} \operatorname{sgn}(\alpha-\beta) \theta_{\alpha, \beta},
$$

as generalisation of (4.13). The compatibility of the coefficients (4.27) with the necessary condition for the CBA solvability [15] can be easily checked. Therefore we may conclude that a subclass of the CBA solvable multi-chain models of [15] defined through the parameter choice (4.27) and (4.28) should also belong to a much richer class of quantum integrable systems. Restricting to the twisting transformation alone, i.e. considering only the nontrivial parameter choice $\hat{\theta} \neq 0$, we get a model with Hamiltonian (4.25) and the correlated hopping term reduced from (4.26) to $h_{j j+1}^{(\alpha)}=e^{i\left(+\sum_{\beta \neq \alpha}\left(\operatorname{sgn}(\alpha-\beta) \theta_{\alpha \beta}\left(n_{j+1(\beta)}+n_{j(\beta)}\right)\right)\right.}$. This case is evidently identical to a subclass of the multi-chain Schulz-Shastry model [18], known as the multi-chain nimimal SchulzShastry model, defined for the only nontrivial choice of their generating function: $A_{\alpha \beta}(0)=$ $A_{\alpha \beta}(-1)=\theta_{\alpha \beta}$. At the same time, similar to the single chain case, this minimal class tallies also with the multi-chain generalization of the Kundu model introduced in [22], where however the interchain couplings $\hat{\theta}$ were restricted only to nearest neighbouring chains. Therefore we see that among the multi-chain Schulz-Shastry models only its minimal subclass exhibits quantum 
integrability and allows sufficient number of mutually commuting higher conserved operators together with the Lax operator and the $R$-matrix given by (4.23) transformed by twisting transformation (4.21) with parameters $\vec{\gamma}=0$.

On the other hand considering a combination of twisting (4.21) and gauge transformation (4.20) by choosing $\hat{s}=\hat{\theta}, \hat{g}=0$ one gets the associated Lax operator from (4.23) as

$$
\begin{aligned}
\tilde{L}_{a j}^{(M) Z}(\theta, \lambda) & =S_{a j}^{(M)}(\hat{\theta}) T_{a j}^{(M)}(\hat{\theta}, 0) L_{a j}^{(M) X Y}(\lambda)\left(S_{a j}^{(M)}\right)^{-1}(\hat{\theta}) T_{a j}^{(M)}(\hat{\theta}, 0) \\
& =e^{2 i \sum_{\alpha<\beta} \theta_{\alpha \beta}\left(n_{a(\alpha)} n_{j(\beta)}\right.}\left(\prod_{\alpha}^{M} L_{a j}^{(\alpha) X Y}(\lambda)\right) e^{\left.-2 i \sum_{\alpha<\beta} \theta_{\alpha \beta} n_{a(\beta)} n_{j(\alpha)}\right)} .
\end{aligned}
$$

We notice immediately that the Lax operator (4.29) is a generalisation of (4.16) and coincides exactly with that of the multi-chain Zvyagin et al model [11, 12]. Therefore the Hamiltonian generated from it should have the form

$$
\tilde{H}^{(M) Z}=-\sum_{j, \alpha}^{N, M} c_{j+1(\alpha)}^{\dagger} c_{j(\alpha)} e^{2 i\left(-\sum_{\beta>\alpha} \theta_{\alpha \beta} n_{j+1(\beta)}+\sum_{\beta<\alpha} \theta_{\alpha \beta} n_{j(\beta)}\right)}+\text { h.c. }
$$

This also establishes, as in the single chain case, that the correct form of the multi-chain Zvyagin et al model should be given by the Hamiltonian (4.30), which is related to the minimal multichain Schulz-Shastry model by an unitary transformation $U_{M}=\exp \left(-i \sum_{j, \alpha \neq \beta} \theta_{\alpha, \beta} n_{j(\alpha)} n_{j(\beta)}\right)$, while the Lax operators of these models are connected through the gauge transformation (4.20) with $\hat{s}=\hat{\theta}$. This therefore resolves completely the controversies raised in all earlier works [17, 18, 15] concerning the multi-chain case. Restricting to $M=1$ one naturally recovers all results related to the single chain case obtained above.

\section{Application of the scheme for generating new inte- grable models}

Using the symmetries of the YBE, as we have shown above, one can generate new solutions of YBE and hence generate new quantum integrable models starting from the old ones. Through this scheme we have already identified the integrable subclass of the CBA solvable models classified earlier. Using other options of the same scheme we intend to generate now new models. Firstly we exploit the freedom in choosing the transformation operator $g_{a}$ (4.6) in any arbitrary form. Note that until now we have chosen these operators from the Cartan generators only ignoring their other choices like

$$
g_{j}^{+}=e^{i \theta^{+} \sigma_{j}^{+} \tau_{j}^{-}}, g_{j}^{-}=e^{i \theta^{-} \sigma_{j}^{-} \tau_{j}^{+}}
$$

which we may use now in transformation (4.6) either individually or in combinations. Without detailed analysis of individual cases we demonstrate here only a sample case of $g^{+}$on a noninteracting two-chain $X X X$ model: $H^{0 x x x}=\sum_{j} H_{j j+1}^{0 x x x(2)}=-\sum_{j} \vec{\sigma}_{j} \vec{\sigma}_{j+1}+\vec{\tau}_{j} \vec{\tau}_{j+1}$. Using only the 
CR (4.5) it can be easily shown that this transformation introduces various interacting terms including interchain hopping as well as pair production terms in the form:

$$
\begin{aligned}
H^{x x x(2)} & =\sum_{j} g_{j}^{+} g_{j+1}^{+}\left(H_{j j+1}^{0 x x x(2)}\right)\left(g_{j}^{+} g_{j+1}^{+}\right)^{-1} \\
& =H^{0 x x x(2)}-\sum_{j}\left[i \theta ^ { + } \left(\left(\sigma_{j}^{+} \tau_{j}^{-}\left(\tau_{j+1}^{3}-\sigma_{j+1}^{3}\right)+\sigma_{j+1}^{+} \tau_{j+1}^{-}\left(\tau_{j}^{3}-\sigma_{j}^{3}\right)\right)-\left(\sigma_{j}^{+} \tau_{j+1}^{-}\left(\tau_{j}^{3}-\sigma_{j+1}^{3}\right)\right.\right.\right. \\
& \left.\left.+\sigma_{j+1}^{+} \tau_{j}^{-}\left(\tau_{j+1}^{3}-\sigma_{j}^{3}\right)\right)+2\left(\theta^{+}\right)^{2} \sigma_{j}^{+} \tau_{j}^{-} \sigma_{j+1}^{+} \tau_{j+1}^{-}\right]
\end{aligned}
$$

We can apply similar transformation involving $g_{a}^{-}$on top of the Hamiltonian (5.2) to restore its hermiticity and generate additional interacting term. Another simpler choice of such gauge transformation, given by $g_{j}^{ \pm}(\sigma)=e^{i \rho^{ \pm} \sigma_{j}^{ \pm}}$and similarly for $\vec{\tau}$-operators, act like some rotation on the spin operators. Therefore the effect of such transformations on each anisotropic $X X Z$ spin chain yields

$$
H^{x x z(2)}=H^{0 x x z(2)}-(1-\Delta)\left(i \rho^{+}\left(\sigma_{j}^{+} \sigma_{j+1}^{3}+\sigma_{j}^{3} \sigma_{j+1}^{+}\right)+2\left(\rho^{+}\right)^{2}\left(\sigma_{j}^{+} \sigma_{j+1}^{+}+(\vec{\sigma} \rightarrow \vec{\tau})\right) .\right.
$$

It is clear that at $\Delta=1$, i.e. for the $X X X$ chain the additional interactions in (5.3) vanish due the the rotational invariance of the model. Note that in spite of the additional interactions appearing in the models like (5.2), (5.3) they remain quantum integrable by construction and the associated Lax operators and R-matrices can be constructed through (4.7) using (4.6). Note that though such transformations of the spin models were known in one or in the other forms, their links with the quantum integrable systems and the solutions of the YBE were perhaps never detected earlier. However such gauge transformations, as we have mentioned, do not affect the eigenvalue solution and therefore the Bethe equations are given in the same form as in the original model.

\subsection{New suppersymmetric t-J models with transformed interac- tions}

We can use the freedom of generating operators (4.1) to construct transformed fermionic, bosonic or spin models using the higher rank groups. Here we shall consider such an application using $g l(1,2)$ for constructing integrable variants of suppersymmetric $t-J$ model with correlated hopping and other interactions along with their explicit Lax operator solutions. As we know the $1 \mathrm{~d}$ supersymmetric $t-J$ model with the constraint on the coupling constants: $J=2 t$ turns out to be an exact quantum integrable system with the $R$-matrix and the Lax operator given as

$$
\check{R}_{a j}(\lambda)=c(\lambda) I+b(\lambda) \tilde{P}_{a j}, \quad L_{a j}^{(t J)}(\lambda)=b(\lambda) I+c(\lambda) \tilde{P}_{a j}
$$

where $b(\lambda)=\frac{\lambda}{\lambda+i}, c(\lambda)=\frac{i}{\lambda+i}$ and $\tilde{P}_{a j}$ is the graded permutation operator formed by the generators of $g l(1,2)[7]$. 
The operators (5.4) satisfy now a graded version of the YBE and the regularity condition and therefore following the arguments of (2.5) the Hamiltonian of this SUSY $t-J$ model takes the form $H=\sum_{j} \tilde{P}_{j j+1}$. Since we are interested in the integrable extension of this t-J model with correlated hopping, we can apply (3.1) on its Lax operator for generating new set of Lax operator and R-matrix solutions of the YBE. We may construct the transforming operators $S, T, G$ in the same form (4.2), (4.3) and (4.6) by using pair of operators from the set $n_{+}, n_{-}, n_{0}$. However due to the constraint $n_{+}+n_{-}+n_{0}=I$ we are left with the only choice of $n_{ \pm}$yielding the transformed $t-J$ model as

$$
\begin{aligned}
\tilde{H} & =\sum_{j}\left(-t \mathcal{P} \sum_{\sigma= \pm} c_{j+1(\sigma)}^{\dagger} c_{j(\sigma)} e^{-2 i \gamma_{(\sigma)}}+c c .\right) \mathcal{P} \\
& +2 t\left(\frac{1}{2} S^{+}{ }_{j} S^{-}{ }_{j+1} k_{j j+1}+c c+S^{3}{ }_{j} S^{3}{ }_{j+1}-\frac{1}{4} n_{j} n_{j+1}\right)+n_{j}+n_{j+1}
\end{aligned}
$$

where $\mathcal{P}$ projects out double occupancies, $\mathbf{S}_{j}$ is the spin operator and $n_{j}=n_{j(+)}+n_{j(-)}$ the total number of electrons on site $j$. Note that in (5.5) the operator parts in the correlated hopping given by the same form as (4.10) involving interactions between $\uparrow$ and $\downarrow$ component electrons vanish due to the constraint on double-occupancy indicated by the projector $\mathcal{P}$. However the operator parts arise as additional interactions in the spin terms given by

$$
k_{j j+1}=e^{-i\left(2(\gamma(+)-\gamma(-))+\sum_{\sigma= \pm}\left(\theta\left(n_{j(\sigma)}+n_{j+1(\sigma)}\right)+(s-g) \sigma\left(n_{j(\sigma)}-n_{j+1(\sigma)}\right)\right)\right.} .
$$

On top of this transformation another one may be considered in the factorised form (4.6) taking the factors as $g_{j}^{ \pm}=e^{i \rho^{ \pm} S_{j}^{ \pm}}$, which is equivalent to a $S U(2)$ rotation. Using the commutation relations like

$$
\left[S^{-}, Q_{-}\right]=\left[S^{-}, Q_{+}^{\dagger}\right]=0,\left[S^{-}, Q_{-}^{\dagger}\right]=Q_{+}^{\dagger},\left[S^{-}, Q_{+}\right]=-Q_{-}
$$

where $Q_{ \pm}=\left(1-n_{j(\mp)}\right) c_{j( \pm)}$, one can show that under such rotations, for example with $g_{j}^{-}$, the twisted hopping part of the SUSY $t-J$ model (5.5) would be transformed to

$$
-t \mathcal{P}\left(\sum_{j, \sigma= \pm} c_{j+1(\sigma)}^{\dagger} c_{j(\sigma)} e^{-2 i \gamma_{\sigma}}+\text { c.c. }+i \rho^{-}\left(c_{j+1(+)}^{\dagger} c_{j(-)}\right)\left(e^{-2 i \gamma_{-}}-e^{-2 i \gamma_{+}}\right)\right) \mathcal{P}
$$

introducing hopping between up and down electrons. Since $\left[S^{-}, n_{(+)}+n_{(-)}\right]=0,\left[S^{-}, n_{(+)}-\right.$ $\left.n_{(-)}\right] \neq 0$, such transformations preserve the total charge but not the spin, though they have no effect on the eigenvalue problem as we have mentioned earlier. Nevertheless such $t-J$ models as well as (5.5) with additional interactions represent integrable systems associated with Lax operator and $R$-matrices obtainable from (3.1) starting with (5.4).

We demonstrate below the application of the ABA method for solving the transformed models on the explicit examples of the Hubbard type as well as the SUSY $t-J$ models with correlated hopping. 


\section{Algebraic Bethe ansatz for correlated hopping models}

In a quantum integrable system the ABA method, which solves the EVP for the entire set of conserved operators including the Hamiltonian by solving the EVP for the transfer matrix, works also with equal success for the transformed systems with correlated hopping as introduced above. However, as we see below, among all the transformations discussed above only the twisting transformations can influence the EVP and yield deformed Bethe equations. Since the applications of the ABA to the twisted model goes almost parallelly to that for the original models, we present here only the main steps of the ABA formulation for the twisted Hubbard (4.9) and the t-J model (5.5).

\subsection{ABA for the extended Hubbard model}

The Lax operator and the $R$-matrix for the $1 \mathrm{~d}$ Hubbard model, which may be given by two coupled free fermionic $R$-matrices of 6 -vertex type, were known for quite some time [5]:

$$
\begin{aligned}
L_{a j}^{(h u b)}\left(\lambda_{a}\right) & =\left(L_{a j}^{(+)}\left(\lambda_{a}\right) \otimes\left(L_{a j}^{(-)}\left(\lambda_{a}\right)\right) \exp \left(h_{a} \sigma_{+a}^{3} \sigma_{-a}^{3}\right)\right. \\
R_{a b}^{(h u b)}\left(\lambda_{a}, \lambda_{b}\right) & =\left(L_{a b}^{(+)}\left(\lambda_{a b}\right) \otimes\left(L_{a b}^{(-)}\left(\lambda_{a b}\right)\right) \cosh h_{a b} \cos \tilde{\lambda}_{a b}\right. \\
& +\left(L_{a b}^{(+)}\left(\tilde{\lambda}_{a b}\right) \otimes\left(L_{a b}^{(-)}\left(\tilde{\lambda}_{a b}\right)\right)\left(\sigma_{+a}^{3} \sigma_{-a}^{3}\right) \sinh h_{a b} \cos \lambda_{a b}\right.
\end{aligned}
$$

where $L^{(+)}$and $L^{(-)}$correspond to 6-vertex free fermionic models and involve two independent spin- $\frac{1}{2}$ operators $\sigma_{(+)}$and $\sigma_{(-)}$respectively, representing two spin-components of the electron. In (6.1) the notations $h_{a b}=h_{a}-h_{b}$, with $\sinh 2 h_{a}=\frac{1}{4} U \sin 2 \lambda_{a}$ and $\lambda_{a b}=\lambda_{a}-\lambda_{b}, \tilde{\lambda}_{a b}=\lambda_{a}+\lambda_{a}$ denoting the dependence on the difference and the sum of the spectral parameters have been used. However, in spite of this fact the explicit ABA formulation of the Hubbard model was discovered only recently 23]. This happened possibly due to somewhat unusual tensorial structure of its $16 \times 16 R$-matrix and the $4 \times 4$ Lax operator. Consequently the monodromy matrix has the form

$$
T(\lambda)=\left(\begin{array}{ccc}
B(\lambda) & \vec{B}(\lambda) & F(\lambda) \\
\vec{C}(\lambda) & \hat{A}(\lambda) & \vec{B}^{*}(\lambda) \\
C(\lambda) & \vec{C}^{*}(\lambda) & D(\lambda)
\end{array}\right)
$$

where $\vec{B}(\lambda)\left(\vec{B}^{*}(\lambda)\right)$ and $\vec{C}(\lambda)\left(\vec{C}^{*}(\lambda)\right)$ are two component vectors representing one-particle creation and anihilation operators, while $\hat{A}(\lambda)$ is a $2 \times 2$ matrix with $A_{12}, A_{21}$ are the corresponding spin excitation operators. The scalar operators $F(\lambda)$ and $C(\lambda)$ on the other hand correspond to two-particle creation and annihilation respectively. The integrability condition here is given by a graded version of the YBE (2.3). The ABA becomes tricky due to the presence of a number of creation/annihilation operators, which should be treated properly for solving the 
eigenvalue problem of the transfer matrix $\tau(\lambda)=s \operatorname{Tr}[\operatorname{T(\lambda )}]$, generated by the supertrace of the monodromy matrix (6.2):

$$
\left[B(\lambda)-\sum_{a=1}^{2} A_{a a}(\lambda)+D(\lambda)\right]\left|\lambda_{1}, \ldots, \lambda_{N} ; \mu_{1}, \ldots, \mu_{N_{\downarrow}}>=\Lambda_{N, N_{\downarrow}}\left(\lambda,\left\{\lambda_{j}\right\},\left\{\mu_{\beta}\right\}\right)\right| \lambda_{1}, \ldots, \lambda_{N} ; \mu_{1}, \ldots, \mu_{N_{\downarrow}}>.
$$

This in turn through expansion of $\ln \Lambda_{N, N_{\downarrow}}(\lambda)$ in powers of $\lambda$, as seen from (2.4), solves the eigenvalue problem simultaneously for all conserved operators including the Hamiltonian of the system, e.g. the energy of the model should be given by $E_{N}=\Lambda_{N, N_{\downarrow}}^{\prime}(0) \Lambda_{N, N_{\downarrow}}^{-1}(0)$. The most important point in solving the eigenvalue problem is to identify a $4 \times 4 R$-matrix of the $X X X$ spin $\frac{1}{2}$-chain hidden inside the $R^{H u b}$-matrix of the Hubbard model, which turns out to be the two-particle scattering matrix $S$ of this system constituted out of the matrix elements of $R^{H u b}$ (6.1): $R_{6,6}=R_{11,11}, R_{7,7}=R_{10,10}, R_{10,7}=R_{7,10}$. We have to adopt here the nested or repeated Bethe ansatz approach, choosing the ferromagnetic vaccuum state as the pseudovacuum $\mid 0>$ of the system, which gives the eigenvalue expression as

$$
\begin{aligned}
\Lambda_{N, N_{\downarrow}}\left(\lambda,\left\{\lambda_{j}\right\},\left\{\mu_{\beta}\right\}\right) & =<0|B(\lambda)| 0>\prod_{j}^{N}\left(\frac{R_{1,1}}{R_{2,5}}\left(\lambda, \lambda_{j}\right)\right)+<0|D(\lambda)| 0>\prod_{j}^{N}\left(\frac{R_{4,13}}{R_{8,14}}\left(\lambda, \lambda_{j}\right)\right) \\
& -\prod_{j}^{N}\left(\frac{R_{6,6}}{R_{2,6}}\left(\lambda, \lambda_{j}\right)\right) \Lambda_{N, N_{\downarrow}}^{(1)}\left(\lambda,\left\{\lambda_{j}\right\},\left\{\mu_{\beta}\right\}\right),
\end{aligned}
$$

where the factor $\Lambda_{N, N_{\downarrow}}^{(1)}$ related to the second step in the nested ABA is given by

$$
\begin{aligned}
\Lambda_{N, N_{\downarrow}}^{(1)}\left(\lambda,\left\{\lambda_{j}\right\},\left\{\mu_{\beta}\right\}\right) & =<0^{(1)}\left|A_{11}\left(\lambda-\lambda_{j}\right)\right| 0^{(1)}>\prod_{\beta}^{N_{\downarrow}}\left(\frac{R_{6,6}}{R_{7,10}}\left(\lambda, \mu_{\beta}\right)\right) \\
& +<0^{(1)}\left|A_{22}\left(\lambda-\lambda_{j}\right)\right| 0^{(1)}>\prod_{\beta}^{N_{\downarrow}}\left(\frac{R_{11,11}}{R_{7,10}}\left(\lambda, \mu_{\beta}\right)\right),
\end{aligned}
$$

where $\mid 0^{(1)}>$ is the nested pseudovacuum with respect to the spin excitations. For identifying the effect of the gauge and twisting transfornations we note that we will be concerned only on the modifications of the diagonal elements of the monodromy matrix $T(\lambda)$ and the elements of the scattering matrix $S$ involved in (6.3) through (6.4) and (6.5). It is however not difficult to check through such explicit expressions that the gauge transformations $G, S$ do not enter in any of these elements and therefore do not have any effect on the eigenvalue formulas and consequently on the Bethe ansatz equations. This is the reason why the Zvyagin et al and the minimal Schulz-Shastry models related through a gauge transformation exhibit same Bethe equations as explained above. We find on the other hand that under the twisting transformation (4.3) the relevant terms that suffer changes are the transfer matrix operators $A_{22}\left(\lambda-\lambda_{j}\right) \mid 0>^{1}=$ $e^{-i 2 \theta N} \prod_{j}^{N} b\left(\lambda-\lambda_{j}\right) \mid 0^{(1)}>$ and the element of the scattering matrix $R_{7,10}(\lambda)=b(\lambda) e^{-2 i \theta}$, where 
$b(\lambda)=\frac{\lambda}{\lambda+U}$. For simplicity we shall consider only the parametr $\theta$ in the twisting transformation putting $\gamma_{( \pm)}=0$. Incorporating these changes in (6.5) we get its modified equation under twisting as

$$
\Lambda_{N, N_{\downarrow}}^{(1)}\left(\lambda,\left\{\lambda_{j}\right\},\left\{\mu_{\alpha}\right\}\right)=e^{2 i \theta N_{\downarrow}} \prod_{\beta}^{N_{\downarrow}} \frac{1}{b\left(\mu_{\beta}-\lambda\right)}+e^{2 i \theta\left(N_{\downarrow}-N\right)} \prod_{j}^{N} b\left(\lambda-\lambda_{j}\right) \prod_{\beta}^{N_{\downarrow}} \frac{1}{b\left(\lambda-\mu_{\beta}\right)} .
$$

Notice that at $\lambda=\lambda_{j}$ and $\mu_{\beta}$ singularities appear in some of the terms in the expressions for the eigenvalues (6.4),(6.6). Therefore demanding the vanishing of residues at this points we can arrive at the Bethe equations for determining the parameters $\left\{\lambda_{j}\right\},\left\{\mu_{\beta}\right\}$. The residue of $\Lambda_{N, N_{\downarrow}}(\lambda)$ at $\lambda=\lambda_{j}$ yields

$$
<0\left|B\left(\lambda_{j}\right)\right| 0>\equiv e^{i p_{j} L}=e^{i 2 \theta N_{\downarrow}} \prod_{\alpha=1}^{N_{\downarrow}} \frac{\mu_{\alpha}-\lambda_{j}+U}{\mu_{\alpha}-\lambda_{j}},
$$

while the same at $\lambda=\mu_{\beta}$ gives

$$
e^{i 2 \theta N} \prod_{j=1}^{N} \frac{\mu_{\alpha}-\lambda_{j}+U}{\mu_{\alpha}-\lambda_{j}}=\prod_{\beta=1}^{N_{\downarrow}} \frac{\mu_{\alpha}-\mu_{\beta}+U}{\mu_{\alpha}-\mu_{\beta}-U},
$$

representing the Bethe equations for the integrable Hubbard model with correlated hopping. Note that for $U \rightarrow \frac{i}{2} U$ the Bethe equations (6.7) and (6.7) coinsides exactly with the CBA results corresponding to the Hubbard model with correlated hopping considered in [12, 13, 14]. Note again that the system of [11, 12] is gauge euivalent to the minimal model of [13, which coincides also with that of [14]. We remind that this equivalence holds at the Lax operator and the application of the ABA level, which does not require explicit form of the Hamiltonian. The equivalence however extends also to their Hamiltonians, provided they are crrectly derived from the Lax operators.

\subsection{ABA of twisted $t-J$ model}

The nested ABA treatment for the twisted Hubbard model presented above, may be carried out in a similar way for the twisted $t-J$ model. Interestingly analogous to the Hubbard model, we also find here that, though the twisting transformation $T$ as well as the gauge transformations $G, S$ considered above contribute in generating the correlated hopping terms in the Hamiltonian, only the former type of transformation affect the ABA treatment and the related Bethe equations. The essential ingredients needed for the ABA application to this system, i.e. the transformed Lax operator and the $R$-matrix are given as in (4.14) with (5.4) leading to the corresponding monodromy matrix in the form

$$
T(\lambda)=\left(\begin{array}{ccc}
A_{11}(\lambda) & A_{12}(\lambda) & B_{1}(\lambda) \\
A_{21}(\lambda) & A_{22}(\lambda) & B_{2}(\lambda) \\
C_{1}(\lambda) & C_{2}(\lambda) & D(\lambda)
\end{array}\right)
$$


Since the ABA steps for this twisted model is close to those for the original supersymmetric $t-J$ model, we will follow the formalism of [7] pointing out only the essential differences under twisting. This supersymmetric model involves the bosonic (B) hole state together with the fermionic (F) electron states and we consider the BFF case for concreteness, where in the first step of nesting $\mid 0>$ represent the hole state, over which $C_{1}, C_{2}\left(B_{1}, B_{2}\right)$ act as the creation (annihilation) operators producing fermionic spin- $\downarrow$ and $\uparrow$ pseudo-particles with charge excitations. Spin excitations appear in the next step with $A_{21}\left(A_{12}\right)$ becoming the creation (annihilation) operators over the vacuum $\mid 0^{(1)}>$ mimicing the $X X X$ model. The conserved quantities are again given by the supertrace

$$
s \operatorname{Tr}[T(\lambda)] \equiv \tau(\lambda)=-\left(A_{11}(\lambda)+A_{22}(\lambda)\right)+D(\lambda) .
$$

The eigenfunctions are constructed as

$$
\left|\left\{\lambda_{j}^{(0)}\right\}_{1}^{M_{0}},\left\{\lambda_{\beta}^{(1)}\right\}_{1}^{M_{1}}>=\prod_{j}^{M_{0}} C_{a_{j}}\left(\lambda_{j}^{(0)}\right)\right| 0>F_{a_{1} \ldots a_{M_{0}}}^{(1)}\left(\lambda_{\beta}^{(1)}\right),
$$

where $F_{a_{1} \ldots a_{M_{0}}}^{(1)}\left(\left\{\lambda_{\beta}^{(1)}\right\}\right)=\prod_{\beta}^{M_{1}} A_{12}\left(\lambda_{\beta}^{(1)} \mid 0>^{(1)} F_{\mathbf{b}}^{\mathbf{a}}\right.$. Therefore for finding out the eigenvalue we have to consider carefully the commutation relations between the elemets of $\tau(\lambda)$ (6.10) and the creation operators forming the eigenvector (6.11) using the matrix relations of the graded YBE. This yields finally the eigenvalue expression

$$
\left.\Lambda_{M_{0}, M_{1}}\left(\lambda,\left\{\lambda_{j}^{(0)}\right\},\left\{\lambda_{\beta}^{(1)}\right\}\right)=b(\lambda)\right)^{L} \prod_{j}^{M_{0}} \frac{1}{b\left(\lambda-\lambda_{j}^{(0)}\right)} \Lambda_{M_{0}, M_{1}}^{(1)}(\lambda)+\prod_{j}^{M_{0}} \frac{1}{b\left(\lambda_{j}^{(0)}-\lambda\right)}
$$

where

$$
\Lambda_{M_{0}, M_{1}}^{(1)}(\lambda)=-\left(\prod_{j}^{M_{0}} b_{+}\left(\lambda-\lambda_{j}^{(0)}\right) \prod_{\beta}^{M_{1}} \frac{1}{b_{-}\left(\lambda_{\beta}^{(1)}-\lambda\right)}+\prod_{j}^{M_{0}}\left(\frac{b\left(\lambda-\lambda_{j}^{(0)}\right)}{b\left(\lambda_{j}^{(0)}-\lambda\right)}\right) \prod_{\beta}^{M_{1}} \frac{1}{b_{-}\left(\lambda-\lambda_{\beta}^{(1)}\right)}\right)
$$

Note that the effect of twisting transformation in the above eigenvalue expressions can be detected in the terms $b_{ \pm}(\lambda)=\frac{\lambda}{\lambda+i} e^{ \pm 2 i \theta}$, where the term $b_{+}$comes from the transfer matrix element, while $b_{-}$is the $R$-matrix element $R_{2,5}$ appearing also in the two-particle scattering matrix. The Bethe equations, as discussed above, can be derived from the analiticity condition of the egenvalues yielding

$$
\begin{aligned}
&\left(b\left(\lambda_{j}^{(0)}\right)^{-L} \equiv e^{i p_{j} L}\right.=e^{i 2 \theta M_{1}} \prod_{\beta=1}^{M_{1}} \frac{\lambda_{j}^{(0)}-\lambda_{\beta}^{(1)}+i}{\lambda_{j}^{(0)}-\lambda_{\beta}^{(1)}} \\
& e^{i 2 \theta M_{0}} \prod_{k=1}^{M_{0}} \frac{\lambda_{\alpha}^{(1)}-\lambda_{k}^{(0)}}{\lambda_{\alpha}^{(1)}-\lambda_{k}^{(0)}-i}=\prod_{\beta=1}^{M_{1}} \frac{\lambda_{\alpha}^{(1)}-\lambda_{\beta}^{(1)}+i}{\lambda_{\alpha}^{(1)}-\lambda_{\beta}^{(1)}-i}
\end{aligned}
$$


Note the similarity between the Bethe equations for the twisted $t-J$ and the Hubbard model. However they do differ due to the different structure of their $R$-matrices and the choice of vacua. The CBA analysis [6] of the twisted t-J model (5.5) is expected to lead also to the same Bethe equations as above, which appeared also in [12].

\section{Concluding Remarks}

We have identified a class transformations including gauge and twisting transformations by exploiting the symmetries of the Yang-Baxter equation, which generates multi-chain integrable systems of correlated hopping electron or spin models with interachain interactions. Comparing our construction with the classification of such CBA solvable models made in [15, we identify an important quantum integrable subclass within this CBA solvable models. The solvable models of [15, which are not covered by our scheme might still show integrability, though possibly they would fall under nonultralocal models [19 not considered here and need seperate investigation. On the other hand our construction can go beyond the models classified in [15] and generate other integrable extentions of the $t-J$ and spin models as we have demonstrated here. We have applied the ABA method to the Hubbard and the SUSY $t-J$ models with correlated hopping, which are integrable models constructed through our scheme. The integrable models allow mutually commuting higher conserved operators and the exact solutions of their eigenvalue problem through the ABA treatment. Our findings shows explicitly that only the twisting transformations need to be incorporated in the ABA equations, while the gauge transformations have no effect on them, though both of these transformations can considerably change the form of the Hamiltonian. Therefore this draws the important conclusion that all models related by gauge transformations share the same ABA equations and the eigenvalues, which is in fact the case with the Zvyagin et al model and the minimal model of Schulz and Shastry. Since our method is based on the transformation of the Lax operator associated with the model and not of the Hamiltonian as done in [15], we can get more general information about such transformations. Starting from this general scheme we are not only able to find the explicit relationship between different models known in the literature at their Lax operator and Hamiltonian level, but also could detect some crucial errors in the derivation of a well known model, which resolves completely the recent controvercies around the equivalence and solvability of some known models.

Application of the present scheme of constructing twisted and gauge transformed quantum integrable systems to other models of physical interest, like spin ladder [9], $t-J$ ladder [10], Bariev model 24] should be interesting problems to carry out. Recall also that the transport property in strongly correlated electron systems is probed through the Drude weight [25], 
which is usually calculated using the twisted boundary condition caused by the parameter $\gamma_{(+)}= \pm \gamma_{(-)}=\phi_{ \pm}$in the twisting transformation like (4.3). Therefore the physical relevance of the other twisting parameter $\theta$ considered here, as well as its possible effect in extending the notion of the Drude weight should be explored [26].

It should be mentioned also that various applications and generalizations of the twisting transformation [20] can be found in [27, 28] and the references therein. The most relevant among them in the present context is [28], where twisting transformations were applied to the supersymmetric $t-J$ and $U$ models for deriving their Hamiltonians as well as different forms of the Bethe ansatz equations modified by such twisting.

Acknowledgment: The author thanks the AvH Foundation of Germany for its support through its Followup Programme and the anonymous referee for pointing out some recent references.

\section{References}

[1] A. Lerda and S. Scuito, B 401 (1993) 613.

G.A. Goldin and D.H. Sharp, Phys. Rev. Lett. 76 (1996) 1183.

E. Dagotto and T.M. Rice, Science 271 (1996) 618.

[2] F. D. M. Hald ane, J. Phys. C 14 (1981) 2535.

P. W. Anderson and Z. Zhau, Phys. Rev. B 37 (1988) 627.

P. W. Anderson, Phys. Rev. Lett. 64 (1990) 1839.

[3] H. Bethe, Z. Phys. 71 (1931) 205.

C. N. Yang and C. P. Yang, Phys. Rev. 150, 321 (1966); 151 (1966) 258.

L.A. Takhtajan and L.D. Faddeev, Russian Math. Surveys 34 (1979) 11.

[4] E. H. Lieb and F. Y. Wu, Phys. Rev. Lett. 20 (1968) 1445.

[5] B. S. Shastry, Phys. Rev. Lett. 56 (1986) 2453 ; J. Stat. Phys. 30 (1988) 57.

M. Shiroshi and M. Wadati, J. Phys. Soc. Jpn. 64, 57 (1995)

[6] S. Sarkar, J. Phys A 24 (1991) 1137.

[7] F. H. L. Eßler and V. E. Korepin, Phys. Rev. B 46 (1992) 9147.

[8] F. H. L. Eßler, V. E. Korepin and K. Schoutens, Phys. Rev. Lett. 68 (1992) 2960.

[9] Y. Wang, Phys. Rev. B 60 (1999) 9236. 
[10] Holger Frahm and Anjan Kundu, J. Phys C 11 (1999) L557.

[11] A. Borovik, S. kulinich, V. Popkov and Yu. Strzhemechnyi, Phys. Lett. A 174 (1991) 407. A. E. Borovik, A. A. Zvyagin, V. Yu. Popkov and Yu. M. Strzhemechnyi, JETP Lett. 55 (1992) 292.

[12] A. A. Zvyagin, Sov. J. Low Temp. Phys. 18 (1992) 723.

[13] H. J. Schulz and B. S. Shastry, Phys. Rev. Lett. 80 (1998) 1924.

[14] Anjan Kundu, Exactly integrable family of generalized Hubbard models with twisted Yangian symmetry, Proc. NEEDS'97 (June 12-28,1997, Chania, Greece); Phys. Lett. A 249 (1998) 126.

[15] A. Osterloh, L. Amico and U. Eckern, Nucl. Phys. B 588 (2000) 531.

[16] L. D. Faddeev, Sov. Sc. Rev. C1 (1980) 107.

[17] A. A. Zvyagin, Phys. Rev. Lett. 82 (1999) 2409.

[18] H. J. Schulz and B. S. Shastry, Phys. Rev. Lett. 82 (1999) 2410.

[19] L. Hlavaty and Anjan Kundu, Int J. Mod. Phys. A 11 (1996) 2143 and the references therein.

[20] N. Reshetikhin, Lett. Math. Phys.20 (1990) 331.

[21] A. A. Zvyagin, private communications ( agreed through email discussion).

[22] Anjan Kundu, J. Math.Phys. 41 (2000) 721.

[23] P. B. Ramos and M. J. Martins, J. Phys. A 30 (1997) L195.

[24] R. Z. Bariev, J.Phys. A 24 (1991) L549, L919.

[25] S. Shastry and B. Sutherland, Phys. Rev. Lett. 65 (1990) 243.

S. Fujimoto and N. Kawakami, J. Phys. A 31 (1998) 465.

X. Zotos, Phys. Rev. Lett. 82 (1999) 1740.

[26] Anjan Kundu, under investigation.

[27] Anjan Kundu and B. Basu-Mallick, J. Phys. A 27 (1994) 3091.

M. R-Monteiro, I Roditi, L. Rodrigues and S. Sciuto, J. Lett. B 354 (1995) 389.

B. Basu-Mallick and Anjan Kundu, Nucl. Phys. B 509 [FS] (1998) 705. 
[28] Angela Foerster, J. Links and I Roditi, J. Phys. A 31 (1998) 687.

Angela Foerster, J. Links, J. Phys. A 34 (2001) 5835 